\title{
Efeitos da agitação mecânica e matéria orgânica na análise granulométrica do solo
}

\author{
Mario Miyazawa $^{1} \&$ Graziela M. de C. Barbosa ${ }^{1}$
}

\begin{abstract}
RESUMO
A parte mineral do solo é classificada conforme a granulometria das partículas em argila, silte e areia (fina e grossa) e sua matéria orgânica pode influir no tamanho e na estabilidade dos agregados. 0 objetivo deste trabalho foi avaliar os processos de agitação na eficiência de dispersão dos agregados do solo e a interferência da matéria orgânica na análise granulométrica do solo. Várias amostras de solo foram coletadas nas profundidades de 0 a $40 \mathrm{~cm}$. Foram determinados: tempo de agitação da mesa agitadora reciprocante, tipo de agitador, diâmetro do recipiente para agitação reciprocante, efeito do abrasivo na agitação e oxidação da matéria orgânica com $\mathrm{H}_{2} \mathrm{O}_{2} 30 \%$ na determinação do teor de argila. Agitação reciprocante com areia grossa foi eficiente na desagregação dos agregados do solo e é indicada para análise em série. A oxidação prévia das substâncias húmicas cimentantes do solo com $\mathrm{H}_{2} \mathrm{O}_{2} 30 \%$ não influiu na fragmentação dos agregados. 0 erro causado pela matéria orgânica do solo no teor de argila pode ser corrigido pelo valor da matéria orgânica determinado por Walkley-Black, dispensando oxidação prévia da amostra com $\mathrm{H}_{2} \mathrm{O}_{2} 30 \%$.
\end{abstract}

Palavras-chave: argila, oxidação da matéria orgânica, dispersão, agregados

\section{Effects of mechanical agitation and soil organic matter on soil particle size analysis}

\begin{abstract}
The mineral part of soil is classified according to size of clay, silt and sand (fine and coarse) particles. The soil organic matter $(\mathrm{OM})$ can influence the size and stability of clay aggregates. The aims of this study were to evaluate shaking processes on the dispersion efficiency of soil aggregates and to evaluate the influence of soil O M on soil particle size analysis. Samples from 0 to $40 \mathrm{~cm}$ depths of several agricultural clay soil were collected. Stirring time on reciprocating shaker, shaker types, diameter of container for reciprocating agitation, effect of abrasive on shaking and oxidation of $\mathrm{OM}$ with $30 \%$ of $\mathrm{H}_{2} \mathrm{O}_{2}$ on clay content determination were evaluated. Reciprocating shaking with coarse sand was efficient in the breakdown of soil aggregates and is suitable for series analysis. Previous oxidation of humic substance in soil with $30 \% \mathrm{H}_{2} \mathrm{O}_{2}$ did not influence the fragmentation of clay aggregates. Error on clay content caused by soil $\mathrm{OM}$ can be corrected by using the value of $\mathrm{OM}$ determined by $\mathrm{W}$ alkley-Black method and does not require prior oxidation of soil sample with $30 \% \mathrm{H}_{2} \mathrm{O}_{2}$.
\end{abstract}

Key words: clay, organic matter oxidation, dispersion, aggregates 


\section{INTRODUÇÃO}

Os solos minerais são constituídos de diferentes tamanhos de partículas e classificados segundo a distribuição das frações: argilas menores que 2,0 $\mu \mathrm{m}$, silte entre 2,0 a 20,0 $\mu \mathrm{m}$ e areias maiores que $20,0 \mu \mathrm{m}$. As argilas são formadas por silicato de $\mathrm{Fe}$ e Al e a unidade dessas partículas forma agregados estáveis de óxidos metálicos e substâncias húmicas. A estabilidade e o tamanho dos agregados aumentam de acordo com o aumento das concentrações de substâncias húmicas, principalmente ácidos húmicos, fúlvicos e polissacalídeos e também com o aumento das concentrações de óxidos e hidróxidos de Fe e de Al (Ferreira et al., 1999; Donagemma et al., 2003; Corá et al., 2009). Os açúcares, aminoácidos e outros compostos solúveis do material vegetal, são decompostos rapidamente por microorganismos do solo e compostos fenólicos, taninos, ligninas e polissacarídeos e apresentam maior resistência (Stevenson, 1982). Esses compostos polimerizam e formam ácidos fúlvicos e húmicos, que atuam como agente cimentante das partículas, formando os agregados do solo (Stevenson, 1982; Goldberg et al., 2000).

Em uma condição definida de solo, o manejo do material vegetal, tipo de argila, clima e pH, o teor da matéria orgânica é diretamente proporcional ao teor da argila, mostrando alta afinidade química da carga negativa da matéria orgânica (grupo carboxílico e fenólico) com a carga positiva da argila $\left(\mathrm{M}^{\mathrm{n}+}\right)$ (Stevenson, 1982; Goldberg et al., 2000).

A análise granulométrica do solo requer energia suficiente para vencer a força de ligação dos agregados (Vitorino et al., 2007) e substâncias húmicas, os quais devem ser fragmentados através do choque mecânico (Seta \& Karathanasis, 1996; Suzuki et al., 2004; Sousa Neto et al., 2009) e adição de $\mathrm{NaOH} 0,1 \mathrm{~mol}$ $\mathrm{L}^{-1}$ ou $\mathrm{Na}_{4} \mathrm{P}_{2} \mathrm{O}_{7} 10 \mathrm{H}_{2} \mathrm{O} 0,1 \mathrm{~mol} \mathrm{~L}^{-1}$ (EMBRAPA, 1997).

$\mathrm{O}$ método mais utilizado se baseia na velocidade de sedimentação das partículas em suspensão aquosa, quando então as partículas maiores decantam rapidamente e as menores permanecem mais tempo em suspensão (EMBRAPA, 1997). Mediante essa metodologia, admite-se que as partículas coletadas em 4 min após agitação na profundidade de $5,0 \mathrm{~cm}$ a $20{ }^{\circ} \mathrm{C}$ sejam de argila + silte, e as que forem coletadas na profundidade de $10,0 \mathrm{~cm}$ após $4 \mathrm{~h}$, sejam as argilas. Normalmente, a areia é calculada pela soma de argila + silte subtraída de $1.000 \mathrm{~g} \mathrm{~kg}^{-1}$ (EMBRAPA, 1997; Ruiz, 2005). Para aumentar a eficiência dos dados obtidos faz-se necessária a análise dos dados analíticos (Chang, 2002; Ruiz, 2005). Outra técnica utilizada é baseada na determinação do peso específico da suspensão, utilizando-se o densímetro (Bouyoucus). A leitura da escala no densímetro corresponde a $\mathrm{g} \mathrm{L}^{-1}$ das partículas em suspensão (EMBRAPA, 1997).

Diferentes agitadores são utilizados para a desagregação dos agregados, tais como: coqueteleira de média rotação, coqueteleira de alta rotação, agitador reciprocante com 150 a 300 rpm, agitador com movimento circular de 200 a 300 rpm e movimento orbital com 15 a $20 \mathrm{rpm}$. O tempo de agitação varia de $15 \mathrm{~min}$ a $16 \mathrm{~h}$, dependendo do agitador (Kilmer \& Alexandre, 1949; Oliveira et al., 2002; Tavares Filho \& Magalhães, 2008).

Para a maioria dos solos brasileiros, o uso de qualquer um desses agitadores é capaz de desagregar as partículas de solo, mas os Latossolos com elevado teor de óxidos e hidróxidos de $\mathrm{Fe}$ e de $\mathrm{Al}$ apresentam alta resistência ao choque mecânico na desagregação. As principais características de desagregação incompleta são: menor valor em argila, maior em silte e maior desvio padrão nos resultados. Grohmann \& Raij (1977) concluíram que a etapa mais importante no processo da determinação granulométrica é a desagregação mecânica e verificaram que o método da agitação com baixa rotação e longo período de tempo, com adição de areia é o mais eficaz para os Latossolos estudados, enquanto Oliveira et al. (2002), verificaram que o agitador horizontal de movimento helicoidal, com adição de $30 \mathrm{~g}$ de areia grossa e agitação de $3 \mathrm{~h}$, promoveu maior dispersão em comparação ao método da coqueteleira.

O objetivo deste trabalho foi avaliar os processos de agitação na eficiência de dispersão dos agregados do solo e a interferência da matéria orgânica na análise granulométrica do solo por gravimetria (método da pipeta).

\section{Material e MÉTOdos}

Amostras de solo foram coletadas nos municípios de Londrina (Latossolo Vermelho Distroférrico - LVdf), Palotina (Latossolo Vermelho Eutrófico - LVe), Cascavel (Latossolo Vermelho Distroférrico - LVdf) e Campo Mourão (Latossolo Vermelho Distrófico - LVd), na profundidade de 0-20 cm. Essas amostras foram secadas em estufa a $60^{\circ} \mathrm{C}$, trituradas e passadas em peneira de 2,0 mm; em seguida, foram utilizadas em diferentes experimentos de laboratório para avaliação do tempo de agitação, tipo de agitadores, largura do recipiente para agitação reciprocante e efeito de oxidação da matéria orgânica na análise granulométrica.

A) Tempo de agitação do agitador reciprocante: Foi avaliado com amostras dos solos LVe e LVdf ; transferiram-se 10,0 g de amostra de terra para frasco plástico de $500 \mathrm{~mL}$, forma de paralelepípedo, adicionados $10 \mathrm{~mL}$ de areia grossa (entre $0,5 \mathrm{e}$ $1,0 \mathrm{~mm}$ ), $100 \mathrm{~mL}$ de $\mathrm{NaOH} 0,05 \mathrm{~mol} \mathrm{~L}^{-1}$ e agitadas durante: 0,5 ; 1,$0 ; 2,0 ; 4,0$ e $8,0 \mathrm{~h}$ no agitador reciprocante com $180 \mathrm{rpm}$, amplitude de $38 \mathrm{~mm}$. A suspensão foi transferida para proveta de $500 \mathrm{~mL}$, passada por peneira de $0,2 \mathrm{~mm}$ e completado o volume com água.

Utilizou-se, para as avaliações, o método da pipeta; $4 \mathrm{~h}$ após a homogeneização foram coletados $10 \mathrm{~mL}$ da suspensão, na profundidade de $10 \mathrm{~cm}$ e transferidos para um becker de 25 $\mathrm{mL}$, secadas em estufa a $105^{\circ} \mathrm{C}$ e determinada a massa seca das partículas. Todas as determinações foram feitas com três repetições.

Como estudo complementar foi avaliado o efeito da agitação sem abrasivo (areia grossa) na eficiência na desagregação das partículas. O tempo de agitação avaliado foi de: $2 ; 3 ; 4$ e $6 \mathrm{~h}$ e comparado com $2 \mathrm{~h}$ de agitação com adição de areia grossa (experimento B - item 1).

B) Tipo de agitador: Foram avaliados os seguintes agitadores:

1) movimento reciprocante com $180 \mathrm{rpm}$ - $10,0 \mathrm{~g}$ de amostra de solo foram transferidos para um frasco plástico de $500 \mathrm{~mL}$, com forma de paralelepípedo, aos quais se adicionaram $10 \mathrm{~mL}$ de areia grossa, $100 \mathrm{~mL}$ de $\mathrm{NaOH} 0,05 \mathrm{~mol} \mathrm{~L}^{-1}$, agitados durante 
$2 \mathrm{~h}$, transferindo-se a suspensão para uma proveta de $500 \mathrm{~mL}$ e se completando o volume com água.

2) movimento circular com $220 \mathrm{rpm}$ - idêntico ao procedimento anterior, em que a diferença está na forma cilíndrica do frasco de $600 \mathrm{~mL}$.

3) coqueteleira com $3.200 \mathrm{rpm}$ - 10,0 g de amostra de solo foram transferidos para um copo de coqueteleira de $500 \mathrm{~mL}$; em seguida, adicionados $250 \mathrm{~mL}$ de $\mathrm{NaOH} 0,02 \mathrm{~mol} \mathrm{~L}^{-1}$, mistura que foi agitada durante 15 min e a suspensão transferida para uma proveta de $500 \mathrm{~mL}$.

4) coqueteleira com $12.500 \mathrm{rpm}$ - todos os procedimentos foram idênticos ao anterior, diferindo apenas a rotação do agitador de $12.500 \mathrm{rpm}$.

5) tubo de centrífuga de $50 \mathrm{~mL}$ - transferiu-se $1,0 \mathrm{~g}$ de amostra de terra para um tubo de centrífuga de $50 \mathrm{~mL}$, adicionando-se $1,0 \mathrm{~mL}$ de areia entre 0,5 e 1,0 mm, 25,0 mL de $\mathrm{NaOH} 0,02 \mathrm{~mol} \mathrm{~L}^{-1}$; a solução foi agitada durante $2 \mathrm{~h}$ com tubo na posição horizontal, com movimento reciprocante a $180 \mathrm{rpm}$ e teve o volume completado com água; $4 \mathrm{~h}$ depois foram pipetados 2,0 $\mathrm{mL}$ de suspensão nas profundidades de 5,0; 7,5 e 10,0 cm e determinada a massa seca das partículas. Os solos avaliados nos diferentes tipos de agitador foram: LVdf, LVe, LVdf e LVd.

C) Largura do recipiente para agitador reciprocante: A largura (diâmetro) dos recipientes metálicos utilizados foi: 28; 38; 47; 67; 97 e $122 \mathrm{~mm}$ em agitador reciprocante de $180 \mathrm{rpm}$ e $38 \mathrm{~mm}$ de amplitude. Foram transferidos $10,0 \mathrm{~g}$ de amostra de solo para os recipientes e adicionados $10 \mathrm{~mL}$ de areia grossa, $10 \mathrm{~mL}$ de $\mathrm{NaOH} 0,5 \mathrm{~mol} \mathrm{~L}^{-1}$, completado até a altura da suspensão préestabelecida com água: $5,0 \mathrm{~cm}$ para $28 \mathrm{~mm}$ diâmetro; $5,5 \mathrm{~cm}$ para $38 \mathrm{~mm}, 6,0 \mathrm{~cm}$ para $47 \mathrm{~mm}, 6,5 \mathrm{~cm}$ para $67 \mathrm{~mm}, 7,0 \mathrm{~cm}$ para $97 \mathrm{~mm}, 7,5 \mathrm{~cm}$ em recipiente de $122 \mathrm{~mm}$, e agitados durante $2 \mathrm{~h}$. Com a diferença na altura da suspensão visou-se obter maior choque mecânico. Os solos utilizados neste estudo foram: LVdf, LVe, LVdf.

D) Oxidação da matéria orgânica com $\mathrm{H}_{2} \mathrm{O}_{2} 30 \%$ : Utilizaramse os seguintes procedimentos:

1) Oxidação da matéria orgânica do solo antes da agitação foram transferidos $50 \mathrm{~g}$ de amostra de solo para becker de 500 mLe adicionados lentamente $\mathrm{H}_{2} \mathrm{O}_{2} 30 \%$ sob aquecimento entre 70 a $80^{\circ} \mathrm{C}$, até cessar o desprendimento de gás $\mathrm{CO}_{2}$. Foram gastos entre 200 a $400 \mathrm{~mL}$ de $\mathrm{H}_{2} \mathrm{O}_{2} 30 \%$. As amostras foram secadas em estufa a $60^{\circ} \mathrm{C}$ e trituradas. Para a agitação da amostra usou-se o agitador reciprocante.

2) Oxidação da matéria orgânica da argila após separação após pesagem da argila da TFSA (terra fina secada ao ar) foi adicionado $\mathrm{H}_{2} \mathrm{O}_{2} 30 \%$ até cessar o desprendimento de $\mathrm{CO}_{2} \mathrm{com}$ aquecimento entre 70 a $80^{\circ} \mathrm{C}$ e as amostras de argila assim processadas foram secadas em estufa a $105^{\circ} \mathrm{C}$ e pesadas. Cada amostra foi avaliada em seis repetições.

3) Comparação da oxidação da matéria orgânica do solo, por Walkley-Black e $\mathrm{H}_{2} \mathrm{O}_{2} 30 \%$ - Amostras de solos de diferentes localidades do Paraná foram coletadas: Arapongas (LVef), Sabaudia (LVef), Ibiporã (LVef), Turvo (LBd), Guarapuava (LBd), Santo Antonio da Platina (PVe), Curiúva (PVad) e Ibaiti (LVd), nas profundidades de $0-10 \mathrm{~cm}$ e $20-40 \mathrm{~cm}$ e preparadas TFSA. Foram determinados os teores da matéria orgânica por WalkleyBlack e a perda de massa por oxidação $\mathrm{H}_{2} \mathrm{O}_{2} 30 \%$. Cada amostra foi avaliada em três repetições, pipetando-se $10 \mathrm{~mL}$ da suspensão de cada proveta em becker de $150 \mathrm{~mL}$, secadas em estufa a $105{ }^{\circ} \mathrm{C}$ e pesadas; após a pesagem da argila, foi adicionada lentamente a $\mathrm{H}_{2} \mathrm{O}_{2} 30 \%$ até cessar o desprendimento de gás carbônico; as amostras assim processadas foram secadas na estufa a $105^{\circ} \mathrm{C}$ e pesadas.

\section{RESULTADOS E DISCUSSÃO}

Os resultados de argila determinados em diferentes tempos de agitação reciprocante (Tabela 1), mostram que os valores encontrados para 2 a 8 h não apresentaram diferença pelo desvio padrão. Desta forma, o tempo de $2 \mathrm{~h}$ de agitação foi suficiente para atingir a desagregação para os Latossolos estudados. Como esses solos (Latossolos) apresentam maior resistência à desagregação mecânica, este tempo deverá ser suficiente para se obter a dispersão dos agregados nos demais solos. Referidos resultados são contrários aos encontrados por Grohmann \& Raij (1977) ao concluírem que a agitação com baixa rotação e longo período de tempo, com adição de areia, é o método mais indicado para os Latossolos.

Tabela 1. Efeito do tempo de agitação na dispersão dos agregados (média \pm desvio padrão) em dois Latossolos na profundidade de $0-20 \mathrm{~cm}$

\begin{tabular}{cccccc}
\hline & \multicolumn{5}{c}{ Tempo de agitação da amostra (h) } \\
\cline { 2 - 6 } Solo & $\mathbf{1 , 0}$ & $\mathbf{1 , 5}$ & $\mathbf{2 , 0}$ & $\mathbf{4 , 0}$ & $\mathbf{8 , 0}$ \\
\cline { 2 - 5 } & \multicolumn{5}{c}{ Argila (g kg-1) } \\
$\mathrm{LVe}^{1}$ & $410 \pm 23,1$ & $558 \pm 14,3$ & $570 \pm 9,8$ & $567 \pm 7,9$ & $571 \pm 8,3$ \\
LVdf $^{-1}$ & $577 \pm 33,7$ & $743 \pm 12,3$ & $760 \pm 8,9$ & $763 \pm 9,3$ & $758 \pm 7,7$ \\
\hline
\end{tabular}

Normalmente, os laboratórios de solo utilizam a agitação reciprocante ou agitador rotatório de Wiegner (60 rpm durante $16 \mathrm{~h}$ ), sem abrasivo, razão pela qual se avaliou a eficiência na desagregação da argila dos solos sem adição de abrasivo, reduzindo os tempos de agitação para 2 a $6 \mathrm{~h}$. Verificou-se que, quando não se utiliza o abrasivo, o tempo para a dispersão da argila se eleva para $6 \mathrm{~h}$. Comparando os resultados no tempo de $2 \mathrm{~h}$ para a dispersão da argila, obtem-se $84 \%$ (LVdf) e $92 \%$ (LVe) sem a utilização do abrasivo (Tabela 2).

Tabela 2. Valores de argila dispersa obtidos com agitação reciprocante (média \pm desvio padrão), sem adição de abrasivo em dois Latossolos, na profundidade de $0-20 \mathrm{~cm}$

\begin{tabular}{lcccc}
\hline & \multicolumn{4}{c}{ Tempo de agitação da amostra (h) } \\
\cline { 2 - 5 } Solo & $\mathbf{2 , 0}$ & $\mathbf{3 , 0}$ & $\mathbf{4 , 0}$ & $\mathbf{6 , 0}$ \\
\cline { 2 - 5 } & \multicolumn{4}{c}{ Argila ( $\mathbf{g ~ k g}^{-1}$ ) } \\
LVdf ${ }^{1}$ & $701 \pm 11,1$ & $743 \pm 9,3$ & $742 \pm 9,8$ & $758 \pm 12,9$ \\
LVe & $475 \pm 12,5$ & $532 \pm 10,7$ & $541 \pm 11,2$ & $562 \pm 8,1$ \\
\hline${ }^{1}$ LVdf - Latossolo Vermelho Distroférrico; LVe - Latossolo Vermelho Eutrófico
\end{tabular}

Os resultados mostram que a adição de areia grossa, partículas entre 0,5 a 1,0 mm, reduz o tempo de agitação para completa fragmentação dos agregados de argila.

$\mathrm{Na}$ Tabela 3 se encontram os teores de argila nas amostras dos solos estudados obtidos por diversos agitadores 
comumente utilizados nos laboratórios de solo. A coqueteleira de $3.200 \mathrm{rpm}$ apresentou os menores valores de argila, devido à baixa rotação e ao tempo insuficiente para a desagregação. Por outro lado, a coqueteleira de $12.500 \mathrm{rpm}$ obteve valores semelhantes aos do agitador reciprocante, indicando que a coqueteleira de alta rotação foi eficiente para desagregação dos agregados de argila, no período de $2 \mathrm{~h}$. Os valores obtidos com agitador de movimento circular foram inferiores aos do movimento reciprocante, indicando a ineficiência desse agitador na fragmentação dos agregados de argila.

Tabela 3. Efeito do tipo de agitadores na dispersão dos agregados (média \pm desvio padrão) utilizando-se Latossolos na profundidade de $0-20 \mathrm{~cm}$

\begin{tabular}{|c|c|c|c|c|}
\hline \multirow{3}{*}{ Solo } & \multicolumn{4}{|c|}{ Tipo de agitador } \\
\hline & A & B & C & D \\
\hline & \multicolumn{4}{|c|}{ Argila $\left(\mathrm{g} \mathrm{kg}^{-1}\right)$} \\
\hline LVdf $^{1}$ & $698 \pm 7,2$ & $454 \pm 28,2$ & $692 \pm 9,1$ & $549 \pm 21,7$ \\
\hline LVd & $757 \pm 5,3$ & $508 \pm 17,4$ & $755 \pm 7,5$ & $572 \pm 19,4$ \\
\hline LVe & $568 \pm 10,5$ & $405 \pm 12,4$ & $564 \pm 11,0$ & $464 \pm 8,7$ \\
\hline LVdf & $762 \pm 7,1$ & $497 \pm 17,4$ & $760 \pm 7,8$ & $594 \pm 19,5$ \\
\hline
\end{tabular}

A - Movimento reciprocante, $180 \mathrm{rpm}, 2 \mathrm{~h}$ de agitação; B - Coqueteleira, $3.200 \mathrm{rpm}, 15 \mathrm{~min}$ de agitação; C - Coqueteleira $12.500 \mathrm{rpm}, 15$ min de agitação; D - Movimento circular, $220 \mathrm{rpm}, 2 \mathrm{~h}$ de agitação.

${ }^{1}$ LVdf - Latossolo Vermelho Distroférrico; LVd - Latossolo Vermelho Distrófico; LVe - Latossolo Vermelho Eutrófico

Esses resultados diferem dos encontrados por Kilmer \& Alexandre (1949), Grohman \& Raij (1977) e Tavares Filho \& Magalhães (2008) que indicam a agitação mecânica lenta (16 h a $30 \mathrm{rpm}$ ) das amostras de solo com adição de abrasivos, como a melhor metodologia para dispersão das argilas, porém esses autores compararam seus estudos com a agitação mecânica rápida (10.000 a $12.000 \mathrm{rpm}$ ) utilizando, no entanto, o tempo de agitação variando de 5 a $20 \mathrm{~min}$. Para solos com alto teor de carbonato de cálcio, Sousa Neto et al. (2009) utilizaram os dispersantes $\mathrm{NaOH}$ $0,1 \mathrm{~mol} \mathrm{~L}^{-1}, \mathrm{NaOH} 1 \mathrm{~mol} \mathrm{~L}^{-1}, \mathrm{Na}_{16} \mathrm{P}_{14} \mathrm{O}_{3} 0,1 \mathrm{~mol} \mathrm{~L}^{-1}$ que não se mostraram eficientes na dispersão da argila e verificaram que não houve diferença entre a agitação mecânica rápida e lenta; entretanto, Suzuki et al. (2004), observaram que não é o tempo de contato do dispersante químico ou bolita que influencia a dispersão das amostras e, sim, o tempo de agitação das amostras.

Na Tabela 4 estão os valores de argila determinados por diferentes dimensões do recipiente, utilizando-se abrasivo. Os menores valores do recipiente de $28 \mathrm{~mm}$ de diâmetro foram resultantes, provavelmente, da falta de distância para produzir choque contra paredes; por outro lado, no recipiente de 122 mm é provável que tais valores sejam devidos à grande distância entre as paredes, dificultando a produção do choque mecânico na suspensão.
A eficiência na desagregação dos solos nos recipientes de 38 a $97 \mathrm{~mm}$ foi semelhante, porém os maiores valores foram obtidos com 47 e $67 \mathrm{~mm}$. A relação da largura do recipiente/amplitude do agitador desses recipientes foi de 1,25 a 1,75 para alcançar maior eficiência na desagregação dos agregados de argila.

Outra questão que envolve o teor de argila é a exigência deste valor nos laudos de análises de fertilidade apresentados às instituições financeiras para classificar o solo conforme sua aptidão agrícola. Esta exigência ocasionou aumento na demanda para os laboratórios de análise de solos e a rapidez na determinação deste teor é indispensável para a rotina dos laboratórios. Desta forma, o uso de tubo de centrífuga de 50 $\mathrm{mL}$ para agitação na dispersão dos agregados do solo facilita o manuseio e permite maior número de amostras.

A Tabela 5 apresenta os valores de argila pipetada em diferentes profundidades da suspensão, após 4 horas de homogeneização. Os valores que se aproximaram dos $10 \mathrm{~cm}$ da agitação reciprocante durante $2 \mathrm{~h}$ em frasco normal (Tabela 2 , A) foram os de 7,5 cm de profundidade, com maiores valores em desvio padrão; entretanto, tal aumento no erro analítico não altera a classificação do solo para fins de aptidão agrícola.

Tabela 5. Valores de argila (média \pm desvio padrão) obtidos em diferentes profundidade da pipetagem na suspensão em tubo de centrífuga de $50 \mathrm{~mL}$ em Latossolos coletados na profundidade de $0-20 \mathrm{~cm}$

\begin{tabular}{rccc}
\hline Prof. & LVdf $^{1}$ - Londrina & LVe - Palotina & LVdf - Cascavel \\
\cline { 2 - 4 }$(\mathbf{c m})$ & \multicolumn{3}{c}{ Argila $\left(\mathbf{g ~ k g}^{-1}\right)$} \\
5,0 & $709 \pm 26,2$ & $508 \pm 39,7$ & $681 \pm 11,3$ \\
7,5 & $753 \pm 25,2$ & $570 \pm 18,6$ & $702 \pm 22,1$ \\
10,0 & $829 \pm 8,0$ & $595 \pm 13,3$ & $723 \pm 21,2$ \\
\hline${ }^{1}$ LVdf - Latossolo Vermelho Distroférrico; & LVe - Latossolo Vermelho Eutrófico
\end{tabular}

A matéria orgânica do solo interfere na análise granulométrica em duas etapas: a primeira, subestimando os valores das partículas obtidas (argila, silte e areia) da TFSA, pois quando se pesa uma amostra de solo para análise, está incluído o peso da MO; e a segunda, quando se pesa a massa de argila da suspensão, está incluído, também, o peso da matéria orgânica, adsorvida na argila, superestimando, consequentemente, os valores da argila.

Normalmente, na análise granulométrica do solo é utilizada agitação mecânica em meio alcalino para fornecer energia suficiente visando quebrar as forças de ligação entre a argila e as substâncias húmicas; desta forma, a oxidação da matéria orgânica do solo com $\mathrm{H}_{2} \mathrm{O}_{2} 30 \%$ pode favorecer a fragmentação dos agregados de argila. A oxidação prévia da matéria orgânica das amostras de solo com $\mathrm{H}_{2} \mathrm{O}_{2} 30 \%$ contribui na diminuição

Tabela 4. Valores de argila (média \pm desvio padrão) determinados em recipientes com diferentes diâmetros utilizados para a agitação das amostras em diferentes Latossolos

\begin{tabular}{lcccccc}
\hline & \multicolumn{5}{c}{ Recipiente (mm) } \\
\cline { 2 - 7 } Solo/Local & $\mathbf{2 8}$ & $\mathbf{3 8}$ & $\mathbf{4 7}$ & $\mathbf{6 7}$ & $\mathbf{9 7}$ & $\mathbf{1 2 2}$ \\
\cline { 2 - 7 } & & \multicolumn{5}{c}{ Argila (g kg $\mathbf{~}^{-\mathbf{1}}$ ) } \\
LVdf / Londrina & $538 \pm 18,8$ & $719 \pm 11,2$ & $745 \pm 8,1$ & $741 \pm 7,9$ & $739 \pm 9,2$ & $635 \pm 13,2$ \\
LVe / Palotina & $411 \pm 15,2$ & $571 \pm 12,1$ & $569 \pm 9,8$ & $574 \pm 10,1$ & $571 \pm 8,8$ & $527 \pm 11,5$ \\
LVdf / Cascavel & $512 \pm 21,1$ & $697 \pm 8,9$ & $701 \pm 7,7$ & $697 \pm 8,9$ & $678 \pm 8,3$ & $577 \pm 14,5$ \\
\hline
\end{tabular}


do erro na pesagem da amostra e na fragmentação dos agregados.

Os resultados (Tabela 6) indicaram que a oxidação prévia da matéria orgânica dos solos estudados com $\mathrm{H}_{2} \mathrm{O}_{2} 30 \%$, aumentou os valores (B) entre 13 a $17 \mathrm{~g} \mathrm{~kg}^{-1}$ em relação a (A); este aumento foi menor que o teor total da MO desses solos determinados pelo método Walkley-Black, entre 25,5 a 42,9 g $\mathrm{kg}^{-1}$, demonstrando que a oxidação da matéria orgânica com $\mathrm{H}_{2} \mathrm{O}_{2} 30 \%$ foi parcial. Para confirmar esta verificação, usou-se a MO residual de algumas amostras de solo (após oxidação com $\mathrm{H}_{2} \mathrm{O}_{2} 30 \%$ ) pelo método Walkley-Black e se encontraram valores entre 1,0 a $5,0 \mathrm{~g} \mathrm{~kg}^{-1}$.

Tabela 6. Efeito da oxidação da matéria orgânica do solo com $\mathrm{H}_{2} \mathrm{O}_{2} 30 \%$ no teor de argila (média \pm desvio padrão) em Latossolos na profundidade de $0-10 \mathrm{~cm}$

\begin{tabular}{lcccc}
\hline \multirow{2}{*}{ Solo/Local } & MO $^{1}$ & A $^{*}$ & B $^{* *}$ & C*** \\
\cline { 3 - 5 } g kg $^{-1}$ & \multicolumn{3}{c}{ Argila $\left(\mathbf{g ~ k g}^{-1}\right)$} \\
LVdf2/Cascavel & 42,9 & $702 \pm 7,1$ & $713 \pm 6,9$ & $678 \pm 7,3$ \\
LVd/C. Mourão & 42,1 & $751 \pm 5,8$ & $761 \pm 4,9$ & $729 \pm 6,1$ \\
LVe/Palotina & 25,5 & $566 \pm 9,5$ & $574 \pm 7,1$ & $551 \pm 10,2$ \\
LVdf/Londrina & 33,6 & $762 \pm 6,9$ & $775 \pm 4,7$ & $751 \pm 7,5$ \\
\hline
\end{tabular}

${ }^{1}$ Matéria orgânica determinada por Walkley-Black. $A^{*}$ ) TFSA, natural; $B^{* *}$ ) oxidação da matéria orgânica na TFSA com $\mathrm{H}_{2} \mathrm{O}_{2} 30 \%$, antes da dispersão; $\mathrm{C}^{* *}$ ) oxidação da matéria orgânica na argila da TFSA com $\mathrm{H}_{2} \mathrm{O}_{2} 30 \%$ (após separação).

${ }^{2}$ LVdf - Latossolo Vermelho Distroférrico; LVd - Latossolo Vermelho Distrófico; LVe - Latossolo Vermelho Eutrófico

O aumento observado nos valores da argila pela oxidação da matéria orgânica do solo com $\mathrm{H}_{2} \mathrm{O}_{2} 30 \%$, antes da agitação, entre 13 a $17 \mathrm{~g} \mathrm{~kg}^{-1}$, resultou da perda de massa da MO oxidada e não do aumento de fragmentação dos agregados pela oxidação da matéria orgânica ligada às partículas de argila, com $\mathrm{H}_{2} \mathrm{O}_{2} 30 \%$.

Os resultados indicam que os agregados de argila dos Latossolos podem ser totalmente fragmentados com agitação mecânica na presença de solução de $\mathrm{NaOH} 0,05 \mathrm{~mol} \mathrm{~L}^{-1}$, dispensando a pré-oxidação da matéria orgânica do solo com $\mathrm{H}_{2} \mathrm{O}_{2} 30 \%$.

Os valores de argila da TFSA (Tabela 6 - item B) estão subestimados, uma vez que, quando se pesa uma amostra de solo para análise, está-se incluído o peso da matéria orgânica e se faz necessária a correção do peso da amostra; por outro lado, quando se pesa a massa de argila da suspensão, aí está incluída a massa da matéria orgânica adsorvida à argila superestimando, em contrapartida, os valores de argila.

Por outro lado, quando se faz a oxidação da matéria orgânica da argila após separação, a quantidade da MO na argila (argila separada da TFSA) oxidada com $\mathrm{H}_{2} \mathrm{O}_{2} 30 \%$ ficou entre 15 a 34 $\mathrm{g} \mathrm{kg}^{-1}$, média de $26 \mathrm{~g} \mathrm{~kg} \mathrm{~kg}^{-1}$, indicando que uma fração das partículas de argila está intimamente ligada às substâncias húmicas (ácidos húmico e fúlvico) do solo, mesmo em alta concentração de $\mathrm{OH}^{-}$, utilizada na dispersão dos agregados de argila (Tabela 6, coluna $\mathrm{C}$ ).

As substâncias húmicas possuem grupos carboxílicos e fenólicos na estrutura molecular. As cargas negativas desses grupos funcionais formam complexos estáveis com metais $\left(\mathrm{Fe}^{\mathrm{n}+}\right.$ e $\mathrm{Al}^{\mathrm{n}+}$ ) da argila com o aumento da concentração de $\mathrm{NaOH}$ na suspensão, desloca os grupos carboxílicos e fenólicos das argilas, haja vista que a estabilidade da ligação M"OH é maior que a da M"carboxílico; no entanto, uma fração permanece como argila-orgânico, em função do equilíbrio químico (Stevenson, 1982; Goldberg et al., 2000). Além disso, como o princípio do método se baseia na velocidade de decantação, as partículas argila-orgânico possuem peso específico menor que as minerais (argilas) e, portanto, permanecerão maior tempo na suspensão, mesmo as partículas maiores que $2,0 \mathrm{~mm}$, causando um erro superestimado nos valores de argila.

Para comparar a oxidação da matéria orgânica da argila com Walkley-Black e $\mathrm{H}_{2} \mathrm{O}_{2} 30 \%$, utilizaram-se alguns solos de municípios paranaenses. Os teores de matéria orgânica determinada por Walkley-Black na TFSA variaram de 8,4 a 66,0 $\mathrm{g} \mathrm{kg}^{-1}$, média de 35,0 $\mathrm{g} \mathrm{kg}^{-1}$ (Tabela 7, coluna A) e os teores de argila variaram de 104 a $803 \mathrm{~g} \mathrm{~kg}^{-1}$ (Tabela 7, colunas B), apresentando ampla variação na textura e nos teores de matéria orgânica do solo.

Tabela 7. Teores de argila da terra fina secada ao ar (TFSA) e argila após oxidação com $\mathrm{H}_{2} \mathrm{O}_{2} 30 \%$, em diferentes tipos de solos paranaenses em duas profundidades

\begin{tabular}{|c|c|c|c|c|c|c|c|}
\hline \multirow{2}{*}{ Solo/Local } & \multirow{2}{*}{$\begin{array}{c}\text { Prof. } \\
\text { cm }\end{array}$} & $\mathrm{MO}^{\mathrm{A}}$ & $\operatorname{Arg}^{B}$ & Arg. $^{C}$ & $\operatorname{Arg}^{\mathrm{D}}$ & Arg. $^{\mathrm{E}}$ & $\mathrm{MO}^{\mathrm{F}}$ \\
\hline & & \multicolumn{6}{|c|}{$\mathrm{g} \mathrm{kg}^{-1}$} \\
\hline \multirow{2}{*}{ LVef $^{1} /$ Arapongas } & $0-10$ & 46,9 & 715 & 750 & 738 & 723 & 36,4 \\
\hline & $20-40$ & 32,1 & 803 & 830 & 825 & 804 & 31,1 \\
\hline \multirow{2}{*}{ LVef/Sabáudia } & $0-10$ & 22,2 & 429 & 439 & 434 & 423 & 37,3 \\
\hline & $20-40$ & 23,1 & 473 & 484 & 480 & 466 & 38,1 \\
\hline \multirow{2}{*}{ LVef/lbiporã } & $0-10$ & 26,2 & 637 & 654 & 648 & 643 & 17,3 \\
\hline & $20-40$ & 20,2 & 677 & 691 & 682 & 681 & 14,8 \\
\hline \multirow{2}{*}{ LBd/Turvo } & $0-10$ & 52,4 & 729 & 769 & 757 & 743 & 34,3 \\
\hline & $20-40$ & 20,0 & 698 & 712 & 708 & 688 & 34,4 \\
\hline \multirow{2}{*}{ LBd/Guarapuava } & $0-10$ & 49,0 & 732 & 770 & 758 & 734 & 46,4 \\
\hline & $20-40$ & 35,0 & 762 & 790 & 783 & 756 & 43,3 \\
\hline \multirow{2}{*}{ PVe/S. A. Platina } & $0-10$ & 20,2 & 121 & 123 & 122 & 115 & 66,1 \\
\hline & $20-40$ & 8,4 & 104 & 105 & 104 & 100 & 48,1 \\
\hline \multirow{2}{*}{ PVAd/Curiúva } & $0-10$ & 66,0 & 585 & 626 & 614 & 581 & 71,8 \\
\hline & $20-40$ & 29,8 & 673 & 694 & 689 & 665 & 41,6 \\
\hline \multirow{2}{*}{ LVd/lbaiti } & $0-10$ & 42,1 & 399 & 417 & 409 & 409 & 20,1 \\
\hline & $20-40$ & 23,3 & 444 & 455 & 451 & 450 & 11,3 \\
\hline
\end{tabular}

(A) Teor da matéria orgânica da TFSA pelo método Walkley-Black; ${ }^{(B)}$ Teor de argila da TFSA (análise de rotina em laboratório); (C) Teor de argila da TFSA corrigida pela massa da MO; (D) MO na TFSA foi oxidada com $\mathrm{H}_{2} \mathrm{O}_{2} 30 \%$, antes da agitação; (E) Teor da argila recalculada da TFSA da coluna ${ }^{(\mathrm{C})}$ com base na MO da argila oxidada com $\mathrm{H}_{2} \mathrm{O}_{2} 30 \%$ após separação; ${ }^{\text {(F) }}$ Teor de MO na argila oxidada com $\mathrm{H}_{2} \mathrm{O}_{2} 30 \%$ (perda da massa)

${ }^{1}$ LVef - Latossolo Vermelho Eutroférrico; LBd - Latossolo Bruno Distrófico; PVe - Argissolo Vermelho Eutrófico; PVAd - Argissolo Vermelho-Amarelo Distrófico; LVd - Latossolo Vermelho Distrófico

A concentração de matéria orgânica na TFSA causa um erro, subestimado na pesagem da amostra de solo; por exemplo, a amostra de LVef (Arapongas) de 0-0,1 m contém 46,9 $\mathrm{g} \mathrm{kg}^{-1}$ de matéria orgânica (Tabela 7, coluna A) e $715 \mathrm{~g} \mathrm{~kg}^{-1}$ de argila (Tabela 7, coluna B); assim, esta quantidade da argila está, na realidade, em 0,95 kg de amostra e não em 1,00 kg. Fazendo a correção e descontando a massa da matéria orgânica na argila da TFSA, ocorreu um aumento entre 10 a $70 \mathrm{~g} \mathrm{~kg}^{-1}$, média de 36 $\mathrm{g} \mathrm{kg}^{-1}$ nos valores de argila; outro erro é a matéria orgânica aderida à argila, pois são substâncias húmicas intimamente ligadas aos metais (Fe, Al e Mn) da argila (Stevenson, 1982). Esses resultados corroboram com Donagemma et al. (2003), em que o pré-tratamento das amostras para oxidação da matéria orgânica e óxidos de ferro e alumínio mal cristalizado, constitui 
uma alternativa eficiente para a completa dispersão das amostras de solo.

A perda de massa de matéria orgânica da argila pela oxidação com $\mathrm{H}_{2} \mathrm{O}_{2} 30 \%$ variou entre 11,3 a 71,8 $\mathrm{g} \mathrm{kg}^{-1}$, média de $36,1 \mathrm{~g}$ $\mathrm{kg}^{-1}$ (Tabela 7, coluna F). Com base nesses valores de perda de massa de matéria orgânica, foram recalculados os valores de argila da TFSA (Tabela 7, coluna C), estando os valores corrigidos na Tabela 7, coluna E.

A oxidação prévia da matéria orgânica na TFSA com $\mathrm{H}_{2} \mathrm{O}_{2}$ $30 \%$ (antes da agitação), causou um aumento de até $50 \mathrm{~g} \mathrm{~kg}^{-1}$, apresentando média de $25 \mathrm{~g} \mathrm{~kg}^{-1}$, nos valores de argila. Dito aumento foi menor do que o teor da argila corrigido utilizandose a massa da matéria orgânica determinada pelo método Walkley-Black, confirmando a ocorrência de oxidação parcial da matéria orgânica do solo com $\mathrm{H}_{2} \mathrm{O}_{2} 30 \%$.

Comparando os valores de argila determinados por dois métodos: teor da argila corrigida pela massa de matéria orgânica determinada pelo método Walkley-Black e oxidação prévia da matéria orgânica com $\mathrm{H}_{2} \mathrm{O}_{2} 30 \%$ (Tabela 6, colunas C e D), a equação da correlação foi: $\mathrm{y}=0,989 \mathrm{x}-0,232 ; \mathrm{R}^{2}=0,9998$, indicando que ambos os métodos podem ser utilizados para oxidação da matéria orgânica na argila do solo, resultados que contradizem os encontrados por Tavares Filho \& Magalhães (2008), que concluíram que a maior dispersão das amostras ocorre quando é realizado o pré-tratamento para oxidação da matéria orgânica; os autores indicam o processo da agitação lenta pela maior eficiência em relação ao processo da agitação rápida.

Em geral, a oxidação da matéria orgânica do solo com $\mathrm{H}_{2} \mathrm{O}_{2}$ $30 \%$ não é total, permanecendo ainda entre 1,0 a $5,0 \mathrm{~g} \mathrm{~kg}^{-1}$ da matéria orgânica residual ou mais, dependendo do tipo de argila. Apesar disso, $\mathrm{H}_{2} \mathrm{O}_{2} 30 \%$ é amplamente utilizado na oxidação da matéria orgânica do solo, visto que não há oxidante prático e de baixo custo, sem destruir a estrutura molecular da argila. A maioria dos oxidantes disponíveis nos laboratórios, tais como: $\mathrm{HClO}_{4}, \mathrm{H}_{2} \mathrm{SO}_{4}, \mathrm{NaClO}$, entre outros, altera a estrutura da argila do solo.

Portanto, visando à análise de solo para fins de fertilidade recomenda-se a correção do teor de argila pelo peso da matéria orgânica do solo determinado por Walkley-Black e não pela oxidação prévia com $\mathrm{H}_{2} \mathrm{O}_{2} 30 \%$, em função da morosidade do processo, inviabilizando as análises de solo em série.

\section{ConclusõES}

1. Agitação reciprocante com areia grossa é eficiente na desagregação dos agregados do solo e indicada para análise em série.

2. A oxidação prévia das substâncias húmicas cimentantes do solo com $\mathrm{H}_{2} \mathrm{O}_{2} 30 \%$ não influiu na fragmentação dos agregados das argilas.

3. O erro causado no teor de argila pela matéria orgânica do solo mineral pode ser corrigido pelo valor da matéria orgânica determinado por Walkley-Black, dispensando oxidação prévia da amostra com $\mathrm{H}_{2} \mathrm{O}_{2} 30 \%$.

\section{LITERATURA CITADA}

Chang, K. L. Optimal estimation of the granulometric composition of soil. Soil Science, v.167, p.135-146, 2002.

Corá, J. E.; Fernandes, C.; Beraldo, J. M. G.; Marcelo, A. V. Adição de areia para dispersão de solos na análise granulométrica. Revista Brasileira de Ciência do Solo, v.33, p.255-262, 2009.

Donagemma, G. K.; Ruiz, H. A.; Fontes, M. P.F.; Her, J. C.; Schaefer, C. E. G. R. Dispersão de Latossolos em resposta à utilização de pré-tratamentos na análise textural. Revista Brasileira de Ciência doSolo, v.27, p.765-772, 2003.

EMBRAPA - Empresa Brasileira de Pesquisa Agropecuária. Manual de métodos de análise de solo. Rio de Janeiro: Centro Nacional de Pesquisa de Solos. 1997. 212p. Documentos, 1

Ferreira, M. M.; Fernandes, B.; Curi, N. Influência da mineralogia da fração argila nas propriedades físicas de Latossolos da região sudeste do Brasil. Revista Brasileira de Ciência do Solo, v.23, p.515-523, 1999.

Goldberg, S.; Lebron, I.; Suarez, D. L. Soil colloidal behavior. In: Summer, M. E. Handbook of soil science. New York: CRC Press, 2000. Cap. 6, p. B195-B240.

Grohmann, F.; Raij, B. van. Dispersão mecânica e pré-tratamento para análise granulométrica de Latossolos Argilosos. Revista Brasileira de Ciência do Solo, v.1, p.52-53, 1977.

Kilmer, J. R.; Alexandre, L. T. Methods of making mechanical analysis of soil. Soil Science, v.68, p.15-24, 1949.

Oliveira, G. C. de; Dias Júnior, M. de S.; Vitorino, A. C. T.; Ferreira, M. M.; Sá, M. A. C. de; Lima, J. M. Agitador horizontal de movimento helicoidal na dispersão mecânica de amostras de três Latossolos do sul e campos das vertentes de minas gerais. Ciência eAgrotecnologia, v.26, p.881-887, 2002.

Ruiz, H. A. Incremento da exatidão da análise granulométrica do solo por meio da coleta da suspensão (silte+argila). Revista Brasileira de Ciência do Solo, v.29. p.297-300, 2005.

Seta, A. K.; Karathanasis, A. D. Water dispersible collids and influencing their dispersibility from soil aggregates. Geoderma, v.74, p. 255-266, 1996.

Sousa Neto, E. L. de; Figueiredo, L. H. A.; Beutler, A. N. Dispersão da fração argila de um Latossolo sob diferentes sistemas de uso e dispersantes. Revista Brasileira de Ciência do solo, v.33. p.723-728, 2009.

Suzuki, L. E. A. S.; Reinert, D. J.; Kaiser, D. R.; Kunz, M.; Pellegrini, A.; Reichert, J. M.; Albuquerque, J. A. Teor de argila de solos sob diferentes tempos de agitação horizontal, tempo de contato do dispersante químico e dispersão mecânica. In: XV Reunião Brasileira de Manejo e Conservação do Solo e da Água, 2004, Santa Maria/RS. Anais...Santa Maria, 2004. CDRom

Stevenson, F. J. Humus chemistry genesis, composition, reaction. New York: John Wiley \& Sons, 1982. 443p.

Tavares Filho, J.; Magalhães, F.S. Dispersão de amostras de Latossolo vermelho eutroférrico influenciadas por prétratamento para oxidação da matéria orgânica e pelo tipo de agitação mecânica. Revista Brasileira de Ciência do Solo, v.32, p.1429-1435, 2008.

Vitorino, A. C. T.; Ferreira, M. M.; Curi, N.; Lima, J. M. D.; Montezano, Z. F. Uso de energia ultra-sônica e turbidimetria na análise textural de pequenas amostras de solo. Revista Ciências Técnicas Agropecuárias, v.16, p.43-48, 2007. 